\title{
ACTIVIDADES PRODUCTIVAS Y VIDA COTIDIANA DE JÓVENES QUE PERMANECEN EN COMUNIDADES INDÍGENAS DE CHIAPAS \\ O QUE HAN MIGRADO AL MEDIO URBANO
}

\section{Productive Activities and Livelihoods OF YOUNGSTERS THAT REMAIN Within Chiapas' INDigenOUS Villages or Have Migrated to Urban Settings}

\section{Raúl Perezgrovas Garza ${ }^{1}$}

Resumen: Para establecer si los jóvenes del medio rural indígena continúan realizando actividades tradicionales, y analizar su vida cotidiana si salen al medio urbano, se llevaron a cabo entrevistas semiestructuradas con 21 personas, tsotsiles o tseltales, originarias de siete municipios indígenas de Chiapas. Se establecieron dos categorías: a) jóvenes con residencia dentro de sus comunidades, b) jóvenes con domicilio en el medio urbano. Los primeros, con escolaridad primaria, se ocupan en actividades agropecuarias y labores domésticas, lo mismo que sus progenitores; establecen que su jornada es pesada, y esperan salir para "mejorar sus condiciones de vida" pero no lo hacen porque dicen no hablar bien el castellano. Quienes han llegado al medio urbano alcanzan 13.7 ańos de estudio y a eso se dedican, y salieron para obtener mayores ingresos; sus progenitores se dedican al campo o al comercio. Los jóvenes que viven en los parajes están dentro de una mayoría que quisiera salir para trabajar, estudiar y vestir distinto. Los jóvenes migrantes se vuelven una minoría que intenta integrarse a su nueva vida en el medio urbano.

\footnotetext{
${ }^{1}$ Instituto de Estudios Indígenas de la Universidad Autónoma de Chiapas. Correo electrónico: rgrovas@unach.mx Fecha de recepción: 0209 16; Fecha de aceptación: 081116.
}

(cc) EY-NC-ND Páginas 136-167. 
Palabras clave: vida rural, migración, vida urbana, indumentaria, escolaridad.

Abstract: Aiming to establish if youngsters from indigenous rural areas do perform or are changing their traditional agricultural activities, and to analyze their livelihoods if they decide to migrate into urban settings, a series of semi-structured interviews were carried on with 21 Tsotsil or Tseltal individuals from seven municipalities in Chiapas Highlands. Results show two categories: a) with residence in their original communities, or b) with residence within urban settings. The first ones, scarcely with elementary education, undertake agricultural activities and domestic chores, the same as their parents; they declare that their daily responsibilities are too hard, and they hope to migrate to "improve their livelihoods" although they have not done so because they feel that cannot speak Spanish properly. Those who have already migrated reach 13.7 years of education and are nowadays attending school, and they left their villages to obtain a larger income; their parents are still involved in agricultural or commerce activities. Youngsters who live in the villages are members of an homogenous majority, but they would like to migrate in order to work, to study or to dress differently. Young migrants become a minority trying to integrate into the new livelihood at the urban world.

Keywords: rural livelihoods, migration, urban livelihoods, clothing, education. 


\section{Introducción}

Con frecuencia observamos el paisaje actual de las comunidades indígenas y campesinas de Los Altos de Chiapas y no dejamos de percibir esa sensación de cambio respecto de lo que nuestros padres vieron hace apenas unas décadas. Las parcelas son cada vez más pequeñas, ya no se utilizan azadones, en el trabajo agrícola se emplean bombas aspersoras y agroquímicos, los rebaños de ganado lanar son pequeños, y las mujeres ya no están en los patios de sus viviendas hilando y tejiendo ropa típica. Algo está sucediendo en el medio rural que implica una transformación del modo de vida campesino y puede ser evidencia de un nuevo paradigma sobre el trabajo agropecuario como base de la subsistencia.

Del mismo modo, un recorrido por las calles, comercios y andadores de la ciudad de San Cristóbal de Las Casas, en el centro montañoso de Chiapas, nos muestra a otro tipo de ciudadano, ya no el ladino o el auténtico coleto, sino el habitante de origen indígena que viste, habla y trata de comportarse como mestizo. ¿Estamos presenciando el resultado de un fenómeno de descampesinización, con el consecuente éxodo rural o migración de corto recorrido? Se presiente que la vida cotidiana tanto en el medio rural como en el medio urbano está siendo objeto de una serie de transformaciones que pueden ser producto de los factores sociales y económicos que caracterizan a la época actual. En ese orden de ideas, el objetivo de esta investigación fue analizar si los jóvenes que se mantienen en el medio rural siguen realizando actividades de tipo agropecuario como parte de su vida cotidiana, si están cambiando su entorno ocupacional y productivo dentro de sus parajes, o si establecen nuevas dinámicas sociales una vez que buscan alternativas de vida en el medio urbano.

En este trabajo se registrará la secuencia de actividades que realizan los jóvenes originarios de comunidades indígenas, que parte de su vida cotidiana dentro de su paraje y cómo cambia ésta al llegar al medio urbano; para efectos de comparación y análisis, se presentan luego las labores cotidianas de la generación que antecede a estos jóvenes. En un apartado posterior se examinan los motivos que pueden tener los jóvenes para quedarse en sus comunidades de origen o salir al medio urbano en busca de oportunidades; se hace énfasis en dos aspectos 
de identidad en los jóvenes, la indumentaria que portan y su nivel educativo. El artículo termina con la prospectiva de los jóvenes para su futuro cercano.

\section{Conceptos teóricos}

El presente estudio tiene que ver con la naturaleza de la actividad cotidiana que tienen las personas y sobre su prospectiva de vida en el corto y mediano plazos, ya sea que vivan en comunidades indígenas o que ya hubieran salido de ellas para dirigirse al medio urbano. En cierto modo, esa salida de las localidades rurales en donde nacieron representa un proceso de descampesinización, el cual se ve influido por una serie de factores socioeconómicos y ambientales que pueden relacionarse con la disminución de las cosechas - a causa de modificaciones en los patrones de precipitación pluvial, es decir, por cambio climático-, con el cambio de uso del suelo agrícola para la construcción de viviendas, la migración o la disminución del ingreso familiar (Pérez et al., 2007). Esta perspectiva del joven indígena que migra por primera vez a la ciudad difiere de otra más referida en la literatura centrada en el joven indígena que vive en la periferia de la ciudad de San Cristóbal y que tiene una interacción distinta con el medio urbano (Betancourt, 1997; Cañas, 2014).

En el caso del proceso migratorio del campo a la ciudad, se han establecido en la literatura otros elementos que lo propician, y que se les conoce como factores de expulsión y de atracción; los primeros tienen que ver con la incapacidad que tienen las personas para satisfacer sus necesidades en sus lugares de residencia, mientras que los últimos se asocian con la esperanza que tiene el migrante de satisfacer necesidades o aspiraciones en su nueva localidad (Arango, 1985). En medio de esta dicotomía entre expulsión y atracción se encuentran la distancia física que las personas tienen que recorrer desde sus comunidades de origen, así como la disponibilidad de ofertas de empleo y opciones educativas en los sitios de atracción.

Es necesario situar esta investigación de forma espacial en el centro comercial y cultural que constituye la ciudad de San Cristóbal de Las Casas, y de manera particular su área central, dejando de lado la 
periferia. El centro histórico de la ciudad es confluencia de culturas y lenguas, de gastronomía y música, de costumbres y modas; es en este sitio donde los sujetos de estudio encuentran un nuevo espacio de socialización; aquí se pierden en un mar de acentos que les permite ser uno más sin llamar la atención. Y hablando de este sujeto de estudio, se encuentra en el centro de varios contextos que subyacen, como son en primer lugar el estrato etario de jóvenes que, por un lado, detentan un modo de vida específico y que realizan lo que Cruz-Salazar (2012: 157) denomina "prácticas juveniles", pero que además pertenece a un grupo étnico indígena que no está enmarcado en las actividades tradicionales del medio rural, es decir, del entorno campesino. En una óptica más académica, este trabajo quiere cumplir con la recomendación de la misma autora en el sentido de que "hacen falta etnografías que diluciden esas transiciones, periodos o procesos juveniles no vividos a la manera occidental" (ibídem), investigaciones que ayudarían a entender el paso de joven a adulto de los indígenas de Chiapas que se encuentran en la interfase de la migración, la educación universitaria y el respeto a los usos y costumbres de los antepasados.

\section{Procesos metodológicos}

La metodología empleada se basó en pláticas informales y entrevistas semiestructuradas, para las cuales se diseñó una guía; los instrumentos se aplicaron a jóvenes dentro de sus comunidades indígenas, o en el medio urbano si ahí estaban residiendo; de este modo, el trabajo de campo se llevó a cabo en lugares distintos pero en un espacio de tiempo limitado a cuatro meses —enero-abril de 2016- En el caso de los jóvenes universitarios, los instrumentos se aplicaron a estudiantes de la Universidad Autónoma de Chiapas (Escuela de Gestión y Autodesarrollo Indígena, EGAI-Unach) y la Universidad Intercultural de Chiapas.

Las guías contemplaronn aspectos de vida cotidiana relacionados con su propia identificación como sujetos pertenecientes a una etnia indígena y sus motivaciones para seguir realizando sus actividades en las comunidades de origen o para salir de ellas; en el caso de que ya hubieran dejado los lugares donde residían. La guía pretendía establecer su vida cotidiana actual, los vínculos que tenían con su familia en las 
comunidades de donde provenían y la forma como desarrollaban su "nueva" vida cotidiana. Un aspecto fundamental para la presente investigación era la descripción detallada de las actividades que constituyen el hacer diario de los entrevistados dentro de su espacio social, esto con el propósito de determinar su carácter rural, si es que tenían relación con los procesos agropecuarios, o bien urbano, si estaban en esta situación.

El trabajo de campo utilizó la técnica de muestreo no probabilístico llamada "bola de nieve", por medio de la cual la persona entrevistada sugiere al próximo sujeto a quien se va a aplicar el instrumento, en este caso la guía de entrevista semi-estructurada. Esta técnica es económica y simple, pero puede resultar en un sesgo debido a que los entrevistados tienden a designar personas que conocen bien y con quienes pueden compartir algunas características (Martínez-Salgado, 2012). Además de las personas pertenecientes a una etnia indígena que se encontraban trabajando en sus parajes rurales, y siguiendo los planteamientos de Cruz-Salazar (2012: 146) sobre el perfil de los entrevistados, el universo muestreado en el medio urbano consistió en personas hablantes de una lengua indígena que corresponden al "joven universitario intercultural” y al joven "indígena urbano", dejando fuera al joven "migrante internacional" debido a que no se presentó el caso.

Se consideró importante registrar la escolaridad de cada uno de los entrevistados, el tiempo que dedican a sus actividades habituales así como los principales problemas que encuentran para llevarlas a cabo. También se les preguntó a los sujetos de estudio si estaban desarrollando actividades distintas a las que realizaban sus padres y otros integrantes de sus familias nucleares o extensas en las localidades de origen, así como los problemas que identificaban para llevarlas a cabo. Una parte significativa de las entrevistas fue preguntarle a las personas sobre su prospectiva de vida, es decir, las actividades que les gustaría hacer en el futuro cercano, y los motivos por los que dichas actividades todavía no se estaban realizando.

Los resultados de las entrevistas semiestructuradas se capturaron en hojas de cálculo de Excel para sistematizarlos y determinar los patrones de respuesta. 


\section{Hallazgos y patrones generales}

El presente artículo toca el tema de la situación ocupacional de hombres y mujeres jóvenes que viven actualmente en comunidades rurales o que ya las han dejado para buscar nuevas oportunidades en otros sitios. El trabajo de campo se realizó con 21 personas: 18 mujeres y dos varones, pertenecientes a las etnias indígenas tsotsil y tseltal de Chiapas, más una muchacha de origen náhuatl oriunda del medio rural del estado de Veracruz; los municipios de origen de las personas que participaron en esta investigación son: Chamula, Chalchihuitán, Huixtán, Larráinzar, San Cristóbal de Las Casas, Tenejapa y Oxchuc, todos ellos en la región montañosa de Los Altos.

El análisis de los resultados sugirió establecer dos categorías de acuerdo con la situación actual de los entrevistados: aquellos que seguían viviendo en sus localidades de origen (Dentro=7) y los que ya las habían dejado para buscar otras oportunidades en sitios distintos (Fuera=14). De esta manera ambivalente se estratifican los resultados obtenidos que se presentan en la Figura 1 y que se analizan a continuación. Las personas que corresponden a la categoría "Dentro" fueron entrevistadas en sus propias comunidades rurales, localizadas en los municipios de San Juan Chamula y San Cristóbal de Las Casas; a los de la categoría "Fuera" se les abordó en su sitio actual de estudio o de trabajo en San Cristóbal de Las Casas.

La edad promedio en cada uno de los grupos fue de 22.5 años para quienes están "Dentro" de su comunidad y de 24.3 años los que ya salieron, por lo que puede considerarse que ambas categorías están integradas en el estrato de "adultos jóvenes" que se encuentran entre los 20 y los 24 años de rango etario (CINU, 2016).

La muestra de personas entrevistadas pertenece a una etnia indígena, en este caso tsotsil (76\%) o tseltal (19\%), aunque se dio el caso de una entrevistada de la etnia náhuatl que cursaba estudios de licenciatura fuera de su localidad de origen, aprovechando las ventajas que en la actualidad confiere la estrategia de movilidad académica, por medio de la cual un alumno puede optar por cursar un semestre de su carrera en otra universidad — nacional o extranjera. 
Figura 1. Resumen de hallazgos para jóvenes que viven Dentro o Fuera de su comunidad de origen.

\begin{tabular}{|c|c|c|}
\hline Indicador & Dentro & Fuera \\
\hline Edad, años & $22.5 \pm 7.4$ & $24.3 \pm 5.1$ \\
\hline Escolaridad, años & 6 & 13.7 \\
\hline Dedicación, horas x día ${ }^{-1}$ & 10.5 & 11.7 \\
\hline Principal actividad EGO & Agrícola y pecuaria & Estudios \\
\hline Principal problema EGO & Animales ponzoñosos & Falta de tiempo \\
\hline $\begin{array}{l}\text { Principal actividad } \\
\text { progenitores }\end{array}$ & Agrícola y pecuaria & $\begin{array}{l}\text { Agrícola y pecuaria, asalaria- } \\
\text { do, comercio, artesanías }\end{array}$ \\
\hline $\begin{array}{l}\text { Principal problema } \\
\text { progenitores }\end{array}$ & Analfabetismo & Analfabetismo \\
\hline A EGO le gustaría & $\begin{array}{l}\text { Tener empleo en la ciudad, } \\
\text { vestir a la usanza occidental, } \\
\text { hablar bien castellano }\end{array}$ & $\begin{array}{c}\text { Trabajar en ONG, hacer } \\
\text { agricultura empresarial, tener } \\
\text { negocio propio }\end{array}$ \\
\hline Porque... & $\begin{array}{c}\text { Mejoraría condiciones } \\
\text { de vida }\end{array}$ & Tendría mayores ingresos \\
\hline Por qué no lo ha hecho & $\begin{array}{c}\text { No habla bien castellano, } \\
\text { no hay ingresos }\end{array}$ & $\begin{array}{c}\text { Falta terminar los estudios, } \\
\text { falta organización, falta } \\
\text { capital }\end{array}$ \\
\hline $\begin{array}{l}\text { En cuánto tiempo podrá } \\
\text { hacerlo }\end{array}$ & 1-2 años & 2-4 años \\
\hline
\end{tabular}

Fuente: Elaboración propia con base en las entrevistas.

En relación con la escolaridad de los entrevistados, aquellos que continúan "Dentro" de sus comunidades han cursado la primaria de manera completa o incompleta (75\%) o culminaron estudios de nivel preparatoria (25\%); por su parte, $86 \%$ de quienes están "Fuera" de sus localidades de origen en la actualidad se encuentra realizando estudios universitarios en diferentes semestres de alguna licenciatura. Lo anterior constituye una variante novedosa a las leyes de la migración, que establecían la atracción que ejercían los grandes centros de comercio y de industria (Arango, 1985), cuando en la presente investigación se observa una tendencia de los migrantes para instalarse en zonas urbanas 
en las que es posible proseguir estudios en los niveles educativos que no se ofrecen en sus comunidades de origen. Esta situación representa una nueva modalidad en el estudio de las poblaciones de origen indígena situadas en el medio urbano, y que hacen referencia a su origen dentro de la periferia misma de la ciudad como resultado de los fenómenos migratorios y de expulsión religiosa de las décadas anteriores (Robledo, 2013), o bien como consecuencia del movimiento armado de 1994 (Cañas, 2014). Además, siguiendo los postulados de Betancourt (1997), la población de estos asentamientos en la periferia de la ciudad no puede considerarse como urbana debido a los vínculos que mantiene con las actividades agrícolas que, aun en pequeña escala, se reproducen de manera cotidiana en pequeños traspatios y con reducidos hatos de ovejas (Rodríguez et al., 2005).

\section{Las actividades cotidianas en el medio rural}

En este apartado se describe la vida cotidiana de las personas que permanecen en el medio rural, ya sea que quieran o no emprender un cambio que las lleve al medio urbano. Esta descripción debe ubicarse dentro del debate de lo que significa en la actualidad ser campesino, que es un concepto que ha evolucionado para abarcar mucho más que la clásica definición de Eric Wolf (1966: 3): “es un pequeño productor agrícola que controla sus medios de producción, que son fundamentalmente la posesión de la tierra, pero que incluye algunos aperos de orden rudimentario". En la actualidad, esa definición es rígida, puesto que las condiciones económicas y sociales se han transformado, generando un entorno cambiante; de acuerdo con los postulados de David Gardner

... el momento que vivimos, de la llamada globalización, subraya la multiplicación de etiquetas que le podemos colgar al campesino: migrante eventual, permanente e internacional, indocumentado, vendedor del sector informal, etcétera [...] Así, de entrada se rompe con la noción de campesino como objeto estático en el tiempo y el espacio, para luego plantear la idea de alguien con muchas facetas y con un comportamiento dinámico (Gardner, 1998: 31). 
Las actividades habituales que refieren las personas que siguen estando en los sitios de origen en el medio rural de Chiapas se relacionan con las actividades agrícolas y pecuarias propias del campo, como la siembra de maíz y frijol y su cuidado a lo largo del ciclo agrícola, el pastoreo cotidiano de ganado lanar, el cuidado de cerdos y aves de traspatio - gallinas, guajolotes y patos- y las diversas labores del hogar que incluyen la recolección y acarreo de combustible — leña—, el lavado de ropa, la preparación de alimentos y la limpieza del lugar. Así es, por ejemplo, un día en la vida de Marcelina — tsotsil de 24 años:

Se levanta a las siete de la mañana, lava ropa, acarrea uno o dos "tercios" de leña a 20 minutos de distancia [entre uno y dos kilómetros]; barre cuando le da tiempo. Su trabajo es cuidar los borregos de la familia en el monte [entre 20 y 30 cabezas], en tres sitios diferentes como a una hora de camino; siembra milpa y calabaza y también cuida y le da de comer a las gallinas. Ocasionalmente hace un poco de trabajo textil artesanal. En su casa hace tortillas, lava trastes y limpia los cuartos. El trabajo más pesado es recoger y acarrear la leńa, a veces diario y a veces cada tercer día... (entrevista con Marcelina N., en una comunidad tsotsil, marzo de 2016).

De acuerdo con los cálculos realizados en localidades rurales de Yucatán, Quiroz-Carranza y Orellana (2010) comentan que para una familia de ocho integrantes, el consumo aproximado de leña alcanza poco más de 16 kilogramos, es decir, un "tercio"; en Los Altos de Chiapas, en el caso de Marcelina, que a veces cargue hasta dos "tercios” de leña, es decir, dos viajes, puede explicar el porqué siempre hay un excedente almacenado en las orillas de su vivienda. Además, conociendo el accidentado paisaje de las montańas tsotsiles, se entiende que ella considere el acarreo de leña como el trabajo más pesado en su jornada.

Además de realizar de manera cotidiana las actividades propias del medio rural, en general se aprecia en las personas de este grupo que vive

\footnotetext{
${ }^{2}$ Unidad de medida que consiste en la cantidad de leña que una persona puede cargar en su espalda con ayuda del mecapal, y que puede alcanzar de 15 a 20 kilogramos. Según QuirozCarranza y Orellana (2010), en localidades rurales de Yucatán, el consumo promedio de leña es de $2.1 \mathrm{~kg} \mathrm{x} \mathrm{día}^{-1}$ habitante ${ }^{-1}$.
} 
dentro de sus comunidades de origen el amplio conocimiento de las particularidades de su modo de vida, y así pueden comentar con lujo de detalles sobre el precio de los animales de traspatio - guajolotes, gallinas, borregos y cerdos_-, los sistemas agropecuarios — pastoreo, uso de esquilmos agrícolas para la alimentación animal-y las diferencias estacionales asociadas, sobre el trabajo artesanal de hilado, tejido y bordado, preparación de comidas y acerca de los recursos naturales de su entorno. En el caso de los hombres, empieza a ser común que se dediquen a rentar su fuerza de trabajo, y pasan el resto del tiempo en labores campesinas.

Un aspecto en el que sí coinciden las personas que no han salido de su comunidad es lo pesada que resulta su jornada cotidiana. Rosa María — tsotsil de 27 años — comenta que "trabaja casi todo el día"; se levanta diariamente a las seis de la mañana, y sus actividades incluyen lavar ropa, limpiar la casa, cocinar, alimentar a las gallinas, colectar y acarrear leña, y también cuida a su madre que está enferma; a las 11 de la mañana sale a pastorear su rebaño de ovejas durante seis a ocho horas.

Además de lo anterior, que no es poco, a falta de varones en su casa tiene que realizar trabajo en la parcela cada cuatro o cinco días: siembra, limpieza de maleza, fertilización, cosecha, desgranado, etc. Esta ocupación agrícola es tan ardua que Rosa María quisiera dejar de hacerla: "es mucho trabajo y no puedo pagar [a] quien lo haga", por eso ha pensado conseguir empleo en la ciudad pues, según ella, es más fácil aprender algún oficio y ganar dinero.

Dentro del grupo de personas que permanecen en sus comunidades de origen, otro factor que genera ilusiones y deseos de salir está asociado a las experiencias de migrantes conocidos, ya sea de personas que salieron incluso del país a trabajar y a ganar dinero, como de parientes cercanos que trabajan en la actualidad en el medio urbano. Así lo comenta Julia — tsotsil de 22 años— al decir que

... varios hombres y mujeres de su comunidad están saliendo a Tuxtla [Gutiérrez], a México y a Estados Unidos. Se ve mucho eso de no querer trabajar más en el campo; la mayoría de las muchachitas ya no quieren 
trabajar la ropa [artesanía de lana], sino comprar pantalones y vestidos (entrevista con Julia N., mayo de 2016).

Los entrevistados que permanecen en los parajes indígenas establecieron en sus respuestas que no sienten una presión por la falta de tiempo para realizar sus actividades, sino que están más preocupados por los riesgos naturales que implica el medio; por ejemplo, Marcelina - tsotsil de 24 años- aclara que le dan mucho miedo "las culebras ponzońosas" que en ocasiones se encuentra durante los recorridos de pastoreo en el monte, las cuales "aparecen por tristeza [de las pastoras]", e igualmente comenta que le disgusta mojarse cuando está lloviendo, aun "cuando lleve un mi nailon" para cubrirse, en los términos coloquiales del lenguaje local. Un hecho significativo para las mujeres que permanecen en el campo, particularmente las que cuidan ovejas, es la percepción de inseguridad que sienten por estar solas durante casi todo el día y por tener que caminar por sitios alejados y solitarios; éste es un hecho relacionado con la frecuente escasez de terrenos comunales propios para el pastoreo de ganado o la recolección de especies útiles, lo que implica que cada vez realicen desplazamientos a sitios más distantes de las viviendas.

También se mencionó, en este grupo que persiste en el medio rural, la existencia de algunos problemas de índole agrícola, como la falta de agua en los terrenos de cultivo — generalmente de temporal-, lo que les impide a los trabajadores del campo el tener buenas cosechas o un producto de calidad para vender. Así, Amalia — tsotsil de 23 añoscomenta que se levanta a las siete de la mañana para realizar labores domésticas, y después se va a la parcela a limpiar de maleza el sembradío de clavel; cada cuatro días cosecha flores para llevar a vender, en manojos de 16 unidades, pero cuando "no hay agua suficiente se queman las flores" y se pierde la venta. Desde el punto de vista sociológico, hay que considerar como factor de retención que las personas que permanecen en sus comunidades de origen de alguna manera siguen perteneciendo a un grupo mayoritario dentro de una etnia indígena, situación que les genera la tranquilidad de vivir en el medio en que nacieron, ya que de manera cotidiana emplean su idioma materno, visten el atuendo típico 
de su comunidad, rodeadas de personas conocidas, muchas de ellas parientes sanguíneos o rituales.

Por otro lado, la situación que vive el agro chiapaneco nos hace reflexionar sobre su principal actor social, el campesino, que en los postulados de Chayanov mantiene a la familia como unidad de trabajo organizada y que se desenvuelve en un sistema pre-capitalista o mercantil simple para la satisfacción de sus necesidades (Diez, 2013), pero que ahora se encuentra dentro de una lógica distinta, dejando la tradicional agricultura familiar — milpa y animales de traspatio en un contexto de subsistencia - para tener que diversificar sus actividades económicas. Esta diversificación es cada día más compleja, abarcando actividades que van más allá de una producción agropecuaria distinta, y que se ubica en el ámbito de la elaboración de artesanías para la venta, de la comercialización de productos y excedentes agropecuarios, de la oferta de la fuerza de trabajo, de la inserción en el mundo global de los teléfonos celulares, las redes sociales y la navegación por Internet. Al decir de Cruz-Salazar (2012: 156), los jóvenes universitarios que provienen de comunidades indígenas "compran celulares, ipods y laptops y cuidan mucho que estos aparatos sean artefactos de moda y de alto precio", lo cual implica querer pertenecer a ese grupo social.

En cierto modo, este fenómeno se relaciona con la llamada "descampesinización” porque desmantela el funcionamiento convencional de la organización familiar y convierte al campesino en un "agente multifuncional" que, de este modo, "consolida una nueva estrategia de sobrevivencia familiar" (Acosta, 2005: 8). También hay que considerar que este nuevo "campesino multifuncional" comienza a incursionar en otras actividades productivas que eventualmente se pueden transformar en su principal ocupación, muchas veces adoptando, por ejemplo en el caso del trabajo artesanal, una producción de tipo comercial que lo hace incorporarse al sistema capitalista, concluyendo así su espacio en el ámbito de la agricultura de subsistencia (Pérez et al., 2007).

\section{La vida cotidiana del migrante en el medio urbano}

En el presente estudio, $86 \%$ de las personas que ya salieron de su comunidad de origen se dedica de manera exclusiva a las actividades 
escolares, o de manera parcial cuando combinan el estudio con el trabajo asalariado, por ejemplo, como empleados de mostrador, o con la elaboración de artesanías para ayudarse con sus gastos; estas personas corresponden al joven "estudiante intercultural" que define Cruz-Salazar (2012). Las ocupaciones cotidianas que reportan las personas de este grupo, tanto mujeres como varones, son diversas y corresponden a nuevos espacios de socialización: hacen deporte, escuchan música, salen con sus amigos, caminan por la ciudad, van "a la plaza". Las personas que salieron de su comunidad pero que no se dedican al estudio, por lo general se ocupan en el trabajo doméstico o se contratan como empleados en alguno de los comercios locales, de modo que a eso se circunscribe su diario acontecer.

Desde el punto de vista étnico, las personas que ya salieron de sus parajes pasan a formar parte de un grupo minoritario, es decir, que sufren un proceso de "aculturación" que las pone frente a un lenguaje distinto, con formas y maneras de vestir diferentes, y bajo usos y costumbres disímbolas a las comunes. Este proceso social de aculturación significa, en los términos señalados por Espín y sus colaboradoras:

... un proceso de cambio de actitudes y comportamientos que ocurren, consciente o inconscientemente, en las personas residentes en sociedades multiculturales o que entran en contacto con una nueva cultura debido a procesos migratorios... (Espín et al., 1998: 229).

Son diversos los cambios sociales y culturales que ocurren cuando las personas salen de sus localidades — generalmente rurales — para ingresar a un medio urbano lejano; sin embargo, en relación con el tiempo que dedican a sus actividades, no existió gran diferencia entre las personas que permanecen dentro de su comunidad y las que ya salieron, habiéndose registrado un promedio general de 11.3 horas por día. Lo que sí fue posible apreciar es que los entrevistados que salieron de sus parajes y viven actualmente en el medio urbano aseguran que sus principales problemas son tener "muchas más actividades" y "mucho menos tiempo" para realizarlas. Lo anterior se puede asociar a que dentro de las ciudades siempre existe una amplia variedad de ocupaciones lúdicas 
que cautivan a sus habitantes, en especial para quienes provienen del medio rural, y que les pueden entretener si tienen el tiempo necesario para ello. Ninguna de las personas que se ubican en este grupo que vive fuera de su comunidad se encuentra realizando actividades de tipo agropecuario más allá de cuidar algunas macetas.

El abandonar las actividades habituales que realizaban cuando tenían menos edad, por lo general asociadas a las labores agropecuarias — trabajar en la milpa, cuidar animales de traspatio — y domésticas — lavar, barrer, cocinar, etc.- - y que son las mismas que sus progenitoras hacían o hacen, significa una marcada diferencia respecto de las actividades habituales que actualmente llevan a cabo en su nueva vida cotidiana.

Además, las personas que viven ahora en el medio urbano, y más si son estudiantes universitarios, tienen opiniones propias y discuten sobre temáticas más globales — cambio climático, desaceleración económica, conservación ambiental, situación política del país, etcetera.

Desde una óptica sociológica y siguiendo los postulados de Espín et al. (1998), los sujetos que se enfrentan a situaciones nuevas después de salir de sus comunidades lo hacen inicialmente al nivel "superficial" cuando aprenden de acontecimientos sociales e históricos de la nueva cultura a la que llegan. No obstante, por lo analizado en las entrevistas del presente estudio, las personas que cambiaron su localidad de origen se establecen con relativa velocidad en el nivel "intermedio", es decir, cuando se aprende y se utiliza el nuevo idioma —espańol—y se empiezan a preferir a los nuevos amigos — casi siempre compañeros de escuela o de empleo_- las diversiones a que tienen acceso — más abundantes y variadas - y en general los ambientes que, en el caso que nos ocupa son, entre otros, la vida nocturna de San Cristóbal de Las Casas, los andadores turísticos, y las diversiones que representan los cines, teatros, espectáculos musicales, galerías, mercados y comercios de la ciudad, propician la pronta adaptación.

A pesar de lo anteriormente mencionado, hay que tener en cuenta que todas estas personas que llegaron al medio urbano y que han tenido que modificar su sistema de vida, no se integran de manera absoluta a las nuevas condiciones, es decir, al nivel "significante" que describen Espín et al. (1998) y que conlleva el aceptar nuevos valores, normas 
sociales y patrones de interacción, lo cual únicamente se puede lograr después de mucho tiempo.

Si recordamos que los sujetos de este grupo son en su amplia mayoría estudiantes universitarios, por la estancia perentoria que eso representa es posible adelantar que a lo largo de ella seguirán siendo parte de la minoría que tiene una lengua materna distinta y que su historia está y estará ligada a la familia que permaneció en el medio rural y a la comunidad de origen, lo que significa que el proceso de aculturación no se dará de forma definitiva.

De acuerdo con las mismas autoras, otro factor decisivo para impedir la aculturación total de los nuevos habitantes de la ciudad es su edad, pues salieron de sus comunidades al terminar sus estudios secundarios, que es el periodo en el cual se establecen vínculos importantes con otros iguales, es decir, con otros sujetos pertenecientes a minorías étnicas recién llegados al medio urbano y que, como microcosmos social, se encargan de reforzar la idea de que son un grupo compacto cuyos integrantes se apoyan mutuamente para conservar una identidad étnica distinta a la mayoritaria.

\section{La generación anterior}

Ante preguntas específicas para contrastar si los entrevistados llevan a cabo actividades diferentes a las de sus padres, y con ello tratar de establecer cuándo se rompe el vínculo entre las ocupaciones de la generación anterior y la presente, en el caso de los progenitores y ancestros de quienes viven dentro de sus comunidades, la mayoría (75\%) fue o sigue siendo gente de campo, dedicada a los cultivos agrícolas y a las labores del hogar, mientras que en menor proporción se ocupan en la actualidad de la elaboración de artesanías o del trabajo asalariado. Esto significa que las nuevas generaciones siguen reproduciendo las figuras sociales de sus antecesores, de quienes toman los roles que son el modelo a seguir, puesto que los resultados evidencian que los padres mantienen una actividad relacionada con las labores agropecuarias, las faenas domésticas tradicionales arriba mencionadas, la elaboración de artesanías —en particular las de tipo textil—y el transporte de personas y mercancías. 
Así lo comenta Juan — tsotsil de 22 años—, quien piensa dedicarse a mantener la parcela familiar — maíz, frijol y calabaza-, pero introduciendo algunas variedades de nuevas de frutas que tengan mayor demanda en los mercados locales.

Algo semejante sucede con las personas que ya han salido de sus comunidades, dado que sus ascendientes directos siguen realizando por lo general actividades agrícolas y pecuarias (57\%), aunque ya se mencionan otras labores asociadas al trabajo asalariado y al comercio en pequeño (19\%). El patrón más común es que las madres y abuelas tienen o tuvieron como responsabilidades cotidianas las correspondientes al campo, como lo menciona Cristina — tsotsil de 21 años- al decir que su mamá "se encarga de todo, es ama de casa, y cuida los animalitos como borregos, guajolotes y pollitos", y que sus hermanas "elaboran trajes tradicionales con lana de borrego", al igual que Sandra - tseltal de 24 años- cuya madre atiende la milpa y también "lava ropa y trastes, limpia la cocina y barre toda la casa”, y sus tías "hacen ropa típica de su comunidad".

Sin embargo, también empieza a ser patente que las generaciones anteriores están igualmente modificando algunos patrones del modo de vida tradicional, y de manera gradual van evolucionando incluso dentro de las comunidades en las que nacieron y viven. Lo anterior fue referido por Julia — tsotsil de 22 años—, quien hace énfasis en que “... las mamás ya no enseñan a sus hijas a tejer y a hacer tortilla; se les hace más fácil comprar [esos productos]".

También se registró el hecho de que las antecesoras de quienes no viven más en el campo se alejan actualmente del patrón asociado al medio rural; por ejemplo, Guadalupe — tsotsil de 22 años— recuerda que su madre antes se dedicaba a trabajar la milpa, recoger leña y acarrear agua, cuidar animales, es decir, a las labores propias de una mujer en el medio rural, pero que ahora se dedica a vender tortillas y tamales, $y$ bordar huipiles tradicionales por pedido. Es una situación semejante a la que comentan Silvia — tseltal de 24 años—o Josefa — tsotsil de 20 años-, cuyas antecesoras directas se ocupaban en las labores agrícolas pero que hoy día trabajan como comerciantes o empleadas. 
Estas consideraciones sobre el dinamismo en que hoy en día viven las personas que solían ser campesinos dedicados a su parcela, tienen no sólo implicaciones económicas evidentes, sino que desde la óptica social les confiere un nuevo estatus porque han dejado la pluriactividad agropecuaria; para entender esto, en los términos planteados por Carton de Grammont,

... en los hogares campesinos el trabajo asalariado ha desplazado la actividad agropecuaria (pluriactividad), además de que los campesinos tienden a ser más pobres que los no campesinos. Concluimos con la necesidad de replantear los conceptos clásicos que utilizamos tanto para analizar el sector agropecuario como el espacio rural, en particular los conceptos de la relación campo-ciudad y del campesinado (Carton de Grammont, 2009: 13).

En relación con esta temática, incluso cuando las personas ya hayan salido de su comunidad, la idea de volver al medio rural en algún momento futuro ya denota la influencia que está teniendo la adición de valor y la moda del emprendimiento como factor de cambio, es decir, que hay una nueva visión de su otrora condición de campesinos. Así, Pascuala — tsotsil de 22 ańos—, quien es estudiante universitaria que no se está preparando profesionalmente para la agronomía, relata que le gustaría en el futuro tener un invernadero para producir fresa, e incluso ya ha comenzado a hacerlo en una escala mínima en su vivienda urbana "con cinco macetitas [llenas] con tierra preparada". Por su parte, algunas personas que dejaron su comunidad de origen para estudiar tópicos no asociados a las labores agropecuarias tienen muy presentes los roles de trabajo rural de sus progenitores, y en algún momento les gustaría volver a ellos; este es el caso de Yessenia — náhuatl de 22 años-, quien reconoce que, aunque su abuela se dedicaba a las labores agrícolas, a sus hermanos menores "ya no les gusta trabajar el campo", pero que a ella le encantaría "sembrar hortalizas y tener una composta" para hacer sus productos más amigables con el ambiente.

Analizando esto en perspectiva, se puede aseverar que las personas que salen de sus localidades no necesariamente están reproduciendo patrones 
observados dentro de su familia consanguínea — padres y abuelos-, sino que son, por decirlo de algún modo, la "primera generación" de migrantes, y que tienen el valor y/o la motivación para intentar algo nuevo en algún lugar distinto. Reafirmando de algún modo lo anterior, para Cristina — tsotsil de 21 ańos— uno de sus propósitos que la hizo decidirse a salir de su comunidad en Los Altos de Chiapas y llegar a la ciudad mestiza fue "porque quiero ser totalmente independiente de mis padres y en lo profesional". Esta aseveración coincide con lo expuesto por Cruz-Salazar (2012: 156) cuando menciona que ir el estudiante intercultural a la universidad representa — para algunos_ "un espacio de liberación tanto del yugo familiar como del comunitario".

\section{Sobre causas y efectos}

¿Cuál es entonces el factor detonante para que los adultos jóvenes salgan de sus comunidades indígenas?; en el presente estudio, se analizan algunos factores que pueden ser considerados para explicar su salida hacia los centros urbanos. Se tiene que considerar de manera prioritaria el factor económico, aunque por supuesto no es el único; la mayoría de las personas que siguen viviendo y trabajando en su comunidad refiere, por un lado, que no existe capital monetario para emprender cualquier otra actividad que les permita "mejorar sus condiciones de vida".

Esta situación de carencias monetarias y materiales se puede identificar en las comunidades de la región montañosa central de Chiapas, cuyo nivel de marginación es, según Conapo (2010), muy alto en 16 de los 18 municipios de la región prioritariamente indígena denominada II Altos, que comprende a todas las localidades chiapanecas incluidas en esta investigación. Pero no todo se relaciona con el móvil económico para dejar los parajes; entre esos otros factores que inducen a los jóvenes a salir de la comunidad también se mencionan el carecer de fluidez en el uso del español como lengua "comercial", o que de plano se es analfabeta y al no saber leer ni escribir se sienten limitados para salir y exponerse a situaciones desconocidas. Ése es el caso específico de $43 \%$ de las madres y hermanas de algunas personas entrevistadas que ya salieron de sus parajes rurales, y que en algún momento fueron factores decisivos para impedirles a ellas que abandonaran sus viviendas 
y localidades, e intentaran salir de su zona de confort y buscar nuevos horizontes. Para quienes se encuentran todavía en sus localidades, son igualmente importantes los aspectos de la vida cotidiana que les incitan a pensar con seriedad en el cambio de actividad, entre ellos el que sea "tan pesado" el trabajo en el campo como para intentar obtener un empleo en el medio urbano; sin embargo, también hay otras situaciones que pudieran parecer triviales para quienes no las viven, como por ejemplo María — tsotsil de 23 años_ quien comenta que quisiera salir de su paraje para vestir a la usanza occidental — pantalones de mezclilla, blusa y zapatillas_, pues "le gustaría ponerse unos pantalones, porque nunca los ha usado".

Los argumentos en el caso de las personas que ya salieron de sus parajes indígenas revelan que algunas de ellas (38\%) lo hicieron igualmente por motivos económicos, ya fuera para tener mayores ingresos o para intentar emprender negocios propios que les dieran solvencia e incluso les permitieran generar empleos para otras personas; esto vuelve a confirmar que son factores económicos los que motivan la expulsión de la gente rural (Arango, 1985).

Sin embargo, en $29 \%$ de las entrevistas también se hace referencia a otros factores de expulsión de sus comunidades, tales como el deseo de las personas de seguir estudiando, el conocer nuevos lugares y el vivir experiencias distintas. Confirmando estas aseveraciones, $86 \%$ de las personas que ya migraron de sus localidades de origen se dedica actualmente a cursar estudios, en su mayoría dentro de programas universitarios, y sólo una menor proporción de ellas (9\%) se ocupa en devengar un salario como empleada en el medio urbano. Este salario siempre es menor a las expectativas de los migrantes; así lo explica Juana — tsotsil de 30 años—, pues "pagan muy poco sus patrones, y no le alcanza para los gastos de la escuela de su hija”, que estudia el nivel primario en la educación pública. Hay que considerar que la migración del campo a la ciudad, que en México tuvo un modelo estable durante varias décadas y que permitía a los campesinos emplearse en el mercado laboral de la ciudad, eventualmente terminó por agotarse "tanto por la escasez de trabajo como por la precariedad de los empleos disponibles", que ha dado como resultado que en la actualidad se produzcan procesos 
migratorios más complejos y a sitios cada vez más distantes (Carton de Grammont, 2009: 16).

\section{Indumentaria}

El contexto teórico de la identidad étnica advierte una problemática al abordar su estudio, pues la terminología es confusa y no delimita cabalmente la que es de tipo cultural, racial, minoritaria, o étnica (Espín et al., 1998). Estas autoras hacen patente que la identidad étnica está asociada a fenómenos de organización social — sentido de pertenencia a la comunidad—, de historia y cultura comunes — valores y símbolos habituales - y de percepción de uno mismo como parte de un grupo mayoritario o minoritario.

En ese sentido, y considerando la indumentaria como parte esencial de la identidad asociada a una cultura común, la vestimenta tradicional o típica se convierte en un símbolo de identidad étnica, y representa "una declaración de comunión con otros"; la persona que se viste de cierta manera — a la moda - está tomando una posición que le facilita el ser parte de un grupo o, por lo contrario, ser excluida de lo que representa una corriente establecida y que se da con frecuencia en el medio urbano (Fernández, 2013). De este modo podemos comprender que las mujeres de comunidad sigan utilizando su ropa típica — nagua, faja, huipil- porque les confiere el sentimiento de ser parte de ese grupo mayoritario que habla una lengua indígena, que viste a la usanza tradicional y que además obedece las costumbres del grupo étnico al que pertenecen. La situación cambia cuando esta misma persona sale de su actividad cotidiana en su comunidad para situarse en un medio extraño en el que podría sentirse como parte del grupo minoritario de "extranjeros". Dentro del nuevo medio urbano en que se desenvuelven, al cambiar de vestimenta, de idioma y de actividad, los migrantes se encuentran y se agrupan para dejar de sentirse "forasteros", conformando minorías que se asocian en la escuela y en la calle - los nuevos espacios sociales-, y cuyos integrantes se protegen entre ellos para sentir la fuerza del "grupo". En estos términos lo expone Claudia Fernández, docente investigadora sobre diseño de vestuario y textiles en Colombia, 
... como artefactos culturales, el vestuario y los diferentes elementos de decorado corporal se convierten en vehículos de expresión, símbolos de identidad y declaraciones de una preferencia estética; nuestros cuerpos vestidos hablan y revelan una cantidad de información sin mediación de las palabras... (Fernández, 2013: 6).

Cuando se trata de estudiantes, incluso al nivel universitario, se les puede ver caminando juntos, haciendo sus tareas en pequeños colectivos y socializando entre sus pares. Por un lado se dicen a ellos mismos: "me visto como ladino, hablo como ladino, ocupo un espacio en el medio urbano, y quiero tratar de integrarme a esa 'mayoría' ", de la que por naturaleza no forman parte. Pero no hay que pensar que exista cierto grado de discriminación hacia estas personas que salieron de sus comunidades y que pretenden formar parte del medio estudiantil urbano; en mi experiencia como docente en EGAI-Unach aprecio un respeto hacia ellas y la intención de establecer relaciones de amistad y de sana convivencia estudiantil en el desarrollo del trabajo académico cotidiano. Sin embargo, también se observa una tendencia para que estos pequeños colectivos escolares se conformen por estudiantes que se identifican por ser de un mismo grupo minoritario en el medio urbano, como también se extiende la asociación en el ámbito extracurricular, reuniéndose para participar en diversas actividades lúdicas y asistir a los frecuentes eventos culturales que se presentan en la ciudad.

Dentro de este entorno, resalta el hecho de que algunas estudiantes que provienen de comunidad deciden no hacer modificaciones a su atuendo tradicional, y así se les observa en las escuelas universitarias de la ciudad portando la vestimenta tradicional de sus comunidades. Éste es un caso menos frecuente y constituye más bien la excepción que la regla, aunque es un fenómeno que cada día está ocurriendo con mayor frecuencia. En el caso de los varones dentro de la muestra, ninguno porta el traje típico de su etnia, y mencionan hacerlo sólo cuando regresan a las fiestas de sus pueblos, por lo que la indumentaria no aparece en su discurso como factor determinante. Por el contrario, las mujeres que quisieron salir de sus comunidades para incorporarse al trabajo asalariado dentro de la ciudad mencionan que uno de los factores que 
consideran para hacerlo es precisamente el querer vestir a la manera occidental, tal vez en un intento por pasar desapercibidas en el medio urbano, o quizás para conocer y experimentar cosas novedosas. Así, Juana — tsotsil de 30 años_ viste con ropa de corte occidental para acudir en la ciudad a su trabajo como empleada doméstica de planta a lo largo de la semana, pero durante el descanso de fin de semana en su comunidad porta el atuendo tradicional de los tsotsiles de Chamula; allá viste al estilo occidental y se desenvuelve en el idioma castellano, pero aquí interactúa con su familia en lengua tsotsil y viste nagua hecha con lana y porta blusas bordadas al estilo de su etnia.

Un indicador conveniente en el presente estudio era saber si los entrevistados usan la misma indumentaria que sus progenitores, lo cual fue más categórico en el caso de las mujeres, porque suelen conservar la vestimenta típica más que los varones, dentro y fuera de las comunidades indígenas. En este ámbito de ideas, todas las estudiantes entrevistadas aseveraron no utilizar la ropa tradicional de sus comunidades de origen mientras viven en el medio urbano, excepto en ocasiones muy especiales, mientras que la mayoría de sus progenitoras sí la portaban si continuaban en sus parajes, pero que no lo hacían si ya vivían en la ciudad.

Por el contrario, tanto las mujeres entrevistadas que permanecen en sus localidades de origen como sus progenitoras, acostumbraban vestirse a la usanza tradicional de sus etnias. Lo anterior puede relacionarse con los ya mencionados factores de identidad étnica que se asocian a la indumentaria (Fernández, 2013), y que se modifican cuando las personas permanecen dentro de una mayoría étnica - muchachas indígenas viviendo dentro de su comunidad-o cuando tienen que volverse una minoría al incorporarse a un medio ajeno como puede ser el urbano - muchachas indígenas viviendo fuera de sus parajes.

Puede pensarse entonces que la indumentaria tiene un mayor peso específico como factor de identidad étnica cuando se asocia con otros factores, como el seguir viviendo dentro de las comunidades y mantener el uso de la lengua materna indígena, además de tener mayor contacto con las tradiciones ancestrales; otra situación acontece en el nuevo entorno social y cultural que existe en los sitios de atracción en los centros urbanos, cuando los migrantes prefieren adoptar un estilo 
de vestir diferente e incluso cambian de lengua cotidiana para tratar de adaptarse más rápido y mejor.

\section{Alfabetismo}

Con la finalidad de tener un contexto general sobre el tema del analfabetismo en México, y particularmente en Chiapas, se citan las cifras emanadas del Censo de Población y Vivienda 2010 (Inegi, 2010), que establecen una media nacional de $8.9 \%$ en mujeres y de $6.3 \%$ en varones, datos que son alentadores en referencia al $17 \%$ de la población analfabeta adulta a nivel mundial. Sin embargo, cuando se toman en cuenta los valores de analfabetismo en Chiapas, se indica que con $18.4 \%$ de la población adulta se convierte en la primera entidad federativa en este rubro, seguida de cerca por Guerrero (17.5\%) y Oaxaca (16.9\%). Y ésas son cifras de personas que no acudieron a la escuela, porque adicionalmente hay que considerar el fenómeno del analfabetismo funcional, que significa que incluso tras algunos años de educación primaria, la persona todavía no es capaz de leer o escribir; este indicador alcanza $24.3 \%$ en adultos mayores de 15 años en Chiapas, entidad que ocupa el primer lugar nacional, varios puntos por arriba de la media nacional (González, 2012).

Ya conociendo los indicadores nacionales y estatales, en el presente estudio se observó que, para las personas que continúan viviendo en sus comunidades de origen, uno de los factores que les limita el intentar salir y dedicarse a otras actividades es el de expresarse bien en el idioma del mundo urbano - castellano- o porque su situación de analfabetas reales o funcionales les impide desarrollarse en un medio que les exige al menos leer y escribir para poder competir en el mercado laboral. En esta investigación, la mitad de las personas entrevistadas que aún permanecen en sus localidades manifestó "que no habla bien el español" o que en definitiva no saben leer y escribir.

Así, tanto Lucía - tsotsil de 26 años- como Marcelina — tsotsil de 24 años - tienen la aspiración de emplearse como trabajadoras domésticas en la ciudad, pero mencionan que su limitación es no saber español o no poder hablarlo bien. Ambas jóvenes tsotsiles aseveran haber concluido la educación primaria y, sin embargo, en su plática se 
puede percibir que pertenecen al grupo de las analfabetas funcionales. El discurso educativo oficial presume de grandes logros, pero en la praxis no se observan los resultados; se habla de la educación intercultural como un proyecto de "importancia latinoamericana" que elimina la exclusión social y favorece la integración, aunque apenas se reconocen las grandes limitantes que significan "la marginación socioeconómica y la fragmentación social” (Jiménez, 2011).

Dentro de la presente investigación, de los entrevistados que ya han salido de sus comunidades, la mayoría domina el castellano de una forma fluida, y casi siempre puede escribir en ese idioma con mayor o menor corrección. Debe tomarse en cuenta que la mayoría de los entrevistados en esta categoría no son habitantes originarios de la ciudad, sino una primera generación de migrantes; estas personas son estudiantes bilingües del nivel superior, por ello tienen que desempeñarse académicamente en una lengua diferente a la materna. En contraste, una de las personas de este grupo es analfabeta, de quien se hablará más adelante. Es de hacer notar que $50 \%$ de las progenitoras de las integrantes del estrato de migrantes es todavía analfabeta e incluso sólo hablan su propio idioma, lo que implica que se está frente a una primera generación de individuos que han salido de sus parajes para insertarse en la vida cotidiana del medio urbano.

Un caso excepcional es el de Juana —analfabeta tsotsil de 30 años-, quien tuvo la necesidad de salir de su paraje rural para buscar empleo en la ciudad debido a problemas familiares: "no fui a estudiar, no fui a la escuela, no sé leer ni escribir". Juana tuvo que aprender a ubicarse y moverse en la ciudad de San Cristóbal de Las Casas - hoy por arriba de los 150,000 habitantes - a pesar de no poder leer los letreros de las calles, y relata las vicisitudes que tuvo que pasar para encontrar un domicilio sin saber lo que decía el papel en el que estaba escrito, y el miedo que le representaba no entender las señales de tránsito cuando tenía que ir al mercado, o acudir a la farmacia para conseguir alguna medicina. Sin embargo, comenta que se sobrepuso a tal circunstancia tan desventajosa, de lo cual ya han pasado varios ańos, y que aun siendo analfabeta en la actualidad ha aprendido a hacer su vida cotidiana, sin dejar de reconocer que por esa situación no puede aspirar a otro empleo mejor pagado. 
Esta situación ya había sido analizada por la Comisión Económica para América Latina y el Caribe (Cepal) al establecer en su estudio sobre los impactos sociales y económicos del analfabetismo que

El analfabeto tiene mayores dificultades de inserción social no sólo a nivel personal — problemas de inclusión social, trabajo precario, altas morbilidades, etc.—, sino también a nivel de su grupo familiar - nutrición, higiene, salud y escolaridad de los hijos, entre otros-, y a la sociedad — pérdidas de productividad, altos costos para el sistema de salud (Cepal, 2009: 8).

En el caso de Juana, incluso como analfabeta ha sabido mantenerse trabajando en la ciudad, pero ella misma reconoce que eso representa una limitante para encontrar un empleo mejor pagado: “... la patrona actual es buena y me ayuda, pero paga poco; por eso quiero buscar otro trabajo donde paguen mejor". Reafirmando esta aseveración, ya la Cepal enunciaba que

Los adultos analfabetos tienen grandes limitaciones de empleabilidad debido a un nivel bajo de conocimientos y de especialización. Ello ocurre porque no han tenido acceso a la educación formal; o porque la abandonaron tempranamente para incorporarse al mercado de trabajo o, finalmente, por la pérdida en el tiempo de la capacidad de leer y escribir (Cepal, 2009: 8).

Juana es consciente de que su analfabetismo resulta un factor que va en su contra, así expresa que "le gustaría ir a la escuela y aprender a leer y escribir, para después trabajar en comunidades indígenas"; ella sabe que al incrementar sus capacidades puede aspirar a un empleo mejor pagado. Lo curioso del caso es que Juana ha intentado acudir a la escuela dentro del sistema de educación para adultos (INEA), pero eventualmente se topa con dificultades para hacerlo con asiduidad, pues su misma patrona se encarga de poner trabas a su iniciativa dándole nuevas responsabilidades que le requieren pasar más tiempo en la vivienda donde labora como empleada doméstica de planta. 
Confirmando la importancia que tiene el saber leer y escribir, así como también el poder expresarse en castellano, la mayoría de las personas que ya salieron de su comunidad de origen se desenvuelven bien cuando leen y escriben, y utilizan el idioma castellano de manera usual dentro de sus actividades cotidianas; la forma gramatical de construir su discurso denota con claridad la influencia de los idiomas de origen maya que constituyen la lengua materna de los integrantes de este grupo, que ahora se desenvuelve en el medio urbano mestizo que habla en español.

\section{Vista al futuro}

Las actividades en que los jóvenes entrevistados quisieran involucrarse en el corto plazo, también se diferencian dependiendo de su situación como migrantes en el medio urbano o como habitantes de sus comunidades de origen. Para los primeros, que ya han sido expuestos a otras experiencias por su calidad de estudiantes universitarios, su visión del futuro se relaciona con las actividades lucrativas a las que aspiran en verse involucrados una vez que concluyan sus estudios. Las prioridades se encuentran, curiosamente, dentro del sector agropecuario pero ya con una marcada perspectiva de tipo empresarial — de preferencia siendo los dueños, dijeron algunos_ o teniendo una ocupación remunerada dentro de alguna organización no gubernamental o del gobierno. Resulta interesante advertir que muchos de ellos siguen pensando en regresar al campo, pero ya con un nuevo enfoque que los aleje de las labores agropecuarias convencionales más ligadas a la subsistencia que a la empresa.

Para los integrantes de este grupo de migrantes, las principales limitaciones que les impiden realizar sus sueños de corto plazo son: la necesidad de terminar los estudios que en la actualidad llevan a cabo, lo cual es fácil de entender cuando se habla con estudiantes, o bien el sentimiento de que las nuevas actividades requieren mejor organización en lo personal —necesitan pensar bien lo que quieren hacer y cómo lo van a abordar- y en lo colectivo — se requiere capital económico y capital social para obtenerlocooperativa o una organización social con fines empresariales. Hay que 
tener presente que los estudiantes que formaron parte del estrato de personas que ya salieron de sus comunidades se ocupan actualmente de cursar estudios de nivel superior ligados al desarrollo comunitario, al turismo sustentable y a la gestión de recursos dentro de las universidades Intercultural de Chiapas y Autónoma de Chiapas; por ello, no es extraño que tengan en su mente un contexto ocupacional relacionado con actividades llevadas a cabo dentro del enfoque neoliberal y dentro de un mundo global.

En este sentido, la idea de tener una empresa que ofrezca bienes o servicios encaja muy bien con la idiosincrasia de estos jóvenes que, por otro lado, estan influenciados por la problemática actual de carencia de fuentes de empleo, desaceleración económica y falta de oportunidades para obtener plazas dentro del gobierno estatal o federal. En cuanto al tiempo estimado para dar inicio a esta nueva fase de su vida, en promedio la mayoría de los entrevistados estima que podrá iniciar esas ocupaciones ideales en menos de dos años, lo que se asocia a la duración pendiente de sus estudios.

Por las consideraciones anteriores, los jóvenes indígenas entrevistados en el medio urbano se convierten en personajes que, según las palabras de Cruz-Salazar (2012: 158) tienen una "nueva identidad" asociada con un mayor repertorio cultural y son "muchachos indígenas modernos" que se alejan del campesino tradicional que fuera su antecesor. En esto coincide María Laura Serrano (2015: 159), al establecer que el joven indígena de San Cristóbal de Las Casas está determinado "por su condición étnica y también por la condición de juventud”, y tiene poca relación directa con sus comunidades de origen, lo cual contribuye a su "desarraigo" del medio rural.

Por otro lado, las personas que continúan viviendo en sus parajes presentan aspiraciones mucho más básicas, como mejorar su dominio de una lengua que no es la materna —el castellano_-, o salir del medio rural para emplearse como asalariadas en la ciudad o para iniciar estudios en algún nivel educativo que no existe en sus comunidades; en este sentido, Julia — tsotsil de 22 años de edad— menciona que “...desde chica he querido estudiar y ser alguien en la vida”. Igualmente básicas son las limitaciones que se anteponen al logro de esos objetivos, como la 
falta de recursos económicos para ir a estudiar — porque hay que pagar vivienda, transporte, útiles escolares, etc.- o porque se carece del dominio apropiado del castellano como lengua mayoritaria de uso social y comercial en la región. Sin embargo, la mitad de los entrevistados de esta categoría no sabe cuándo podría dar comienzo a esta nueva vida, aunque percibe que podría ser en el corto aliento — de uno a dos años.

\section{Conclusiones}

Los resultados del trabajo muestran la tendencia de salida de los jóvenes que viven en el medio rural para dirigirse hacia centros urbanos; estos desplazamientos no fueron a grandes distancias, sino a municipios cercanos en donde pudieran trabajar o estudiar. En este trabajo se entrevistó a jóvenes de la primera generación de migrantes desde comunidades rurales, y no desde las colonias periféricas de la ciudad. Los motivos para dejar la comunidad indígena se relacionan con las aspiraciones económicas que resultan de la insuficiencia de ingresos durante su vida en los parajes, lo cual tiene un carácter multifacético en el que destaca el no querer dedicarse a las labores agropecuarias tradicionales que realizan sus ascendientes, y que se puede asociar al fenómeno de descampesinización. Estas manifestaciones contrastan con sus propias expectativas en el mediano plazo, que son regresar a sus comunidades pero con una visión de tipo empresarial dentro de la agricultura o como personal de organizaciones civiles o gubernamentales.

Para los jóvenes que permanecen en sus comunidades de origen, las motivaciones inmediatas son mejorar sus condiciones de vida, conseguir un empleo remunerado y vestir mejor, aunque establecen como limitante el no hablar bien el castellano. Es posible que estas tendencias que tienen los jóvenes de salir de las comunidades indígenas se sigan reproduciendo en tanto el trabajo agropecuario tradicional no genere un ingreso suficiente para el sostén de las familias. 


\section{Bibliografía citada}

Acosta Reveles, Irma Lorena, 2005, "De campesinos a 'multifuncionales'. La explotación agrícola familiar en México”, Revista Vinculo Jurídico, vol. 61, enero-marzo, pp. 38-48.

Arango, Joaquín, 1985, "Las 'leyes de las migraciones' de E. Ravenstein, cien años después", REIS, vol. 32, pp. 7-26.

Betancourt, Darío, 1997, Bases regionales en la formación de comunas rurales-urbanas en San Cristóbal de Las Casas, Chiapas, Universidad Autónoma de Chiapas, Tuxtla Gutiérrez, Chiapas.

Cañas Cuevas, Sandra, 2014, Entre Pueblo Mágico y Ciudad Multicultural: Ciudadanias diversas en la periferia urbana de San Cristóbal de Las Casas, Chiapas, Tesis doctoral, University of Texas at Austin.

Carton de Grammont, Hubert, 2009, "La desagrarización del campo mexicano", Convergencia, Revista de Ciencias Sociales, vol. 50, pp. 13-55.

Cepal (Comisión Económica para América Latina y el Caribe), 2009, Impacto social y económico del analfabetismo: modelo de análisis y estudio piloto, Oficina Regional de Educación, Cepal.

CINU, 2016, Centro de Información de las Naciones Unidas, [en línea] disponible en http://www.cinu.mx/ [consultado el 2 de julio de 2016].

Conapo, 2010, "Índice de marginación por entidad federativa y por municipio 2010. Mapa B.7.2. Chiapas. Lámina 88”, Consejo Nacional de Población, Secretaría de Gobernación, Gobierno Federal, [en línea] disponible en http://www.conapo.gob.mx/ work/models/CONAPO/indices_margina/mf2010/CapitulosPDF/Anexo\%20B2.pdf [consultado el 19 de junio de 2016].

Cruz-Salazar, Tania, 2012, "El joven indígena en Chiapas: el re-conocimiento de un sujeto histórico", Revista LiminaR, vol. X, núm. 2, pp. 145-162.

Diez, María Carolina, 2013, "Campesinado: definiciones analíticas y contextos históricos”, Estudios Rurales, vol. 4, primer semestre 2013, pp. 153-167. 
Espín, Julia, María de los Ángeles Marín, Mercedes Rodríguez y Flor Cabrera, 1998, "Elaboración de un cuestionario para medir la identidad étnica y la aculturación en la adolescencia", Revista de Educación, vol. 315, pp. 227-249.

Fernández Silva, Claudia, 2013, "El vestido como identidad, del gesto personal al colectivo", en C. Fernández, De vestidos y cuerpos, UPB, Medellín, Colombia.

Gardner, David Skerritt, 1998, "Campesinos: ¿de qué hablamos?” Cuadernos de Trabajo núm. 5, Instituto de Investigaciones Histórico-Sociales, Universidad Veracruzana.

González, Norelly, 2012, "Porcentajes de analfabetismo en México", az Revista de educación y Cultura, vol. 63, noviembre, pp. 1-6, [en línea] disponible en http://www.educacionyculturaaz.com/ educacion/porcentajes-de-en-mexico-analfabetismo [consultado el 19 de junio de 2016].

Inegi, Instituto Nacional de Estadística y Geografía, 2010, “Censo de Población y Vivienda 2010. Resultados definitivos”, [en línea] disponible en http://www.inegi.org.mx/est/contenidos/proyectos/ccpv/cpv2010/ [consultado el 15 de junio de 2016].

Jiménez Naranjo, Yolanda, 2011, "Exclusión, asimilación, integración, pluralismo cultural y 'modernización' en el sistema educativo mexicano: un acercamiento histórico a las escuelas de educación pública para indígenas", Revista de Investigación Educativa, vol. 12, enero-junio, Universidad Veracruzana, [en línea] disponible en https://www.uv.mx/cpue/num12/inves/completos/jimenezexclusion-asimilacion.html [consultado el 15 de junio de 2016].

Martínez-Salgado, Carolina, 2012, "El muestreo en investigación cualitativa. Principios básicos y algunas controversias", Ciência \& Saúde Coletiva, vol. 17, núm. 3, pp. 613-619.

Pérez, José Isabel Juan, Samuel Rebollar y José Francisco Monroy Gaytán, 2007, "Descampesinización de una comunidad indígena campesina en México", Observatorio de la Economía Latinoamericana, vol. 75, [en línea] disponible en http://www. eumed.net/cursecon/ecolat/mx/2007/prm.htm [consultado 16 junio de 2016]. 
Quiroz-Carranza, Joaquín y Roger Orellana, 2010, “Uso y manejo de leña combustible en viviendas de seis localidades de Yucatán, México", Madera y Bosques, vol. 16, núm. 2, Xalapa, enero, [en línea] disponible en http://www.scielo.org.mx/scielo. php?script $=$ sci_arttext $\&$ pid $=$ S1 405-04712010000200004 [consultado el 10 de junio de 2016].

Robledo, Gabriela, 2013, "Religión y ciudadanía étnica en Chiapas", Revista SENDAS, vol. 1, pp. 55-73, [en línea] disponible en http://recursosbiblio.url.edu.gt/publicjlg/Rev/Sendas/2013. pdf [consultado el 331 de octubre de 2016].

Rodríguez, Guadalupe, Raúl Perezgrovas, Lourdes Zaragoza, Guadalupe Sánchez y Kokób De Jesús, 2005, "Situación del sistema agropecuario en el contexto rural y urbano en Los Altos de Chiapas", Memorias VI Simposio Iberoamericano sobre Conservación y Utilización de Recursos Zoogenéticos, Cyted-Unach, San Cristóbal de Las Casas, Chiapas, pp. 317-320.

Serrano Santos, María Laura, 2015, “Soy de los dos lados, a la mitad me quedo, Estilos de vida en jóvenes indígenas urbanos de San Cristóbal de las Casas, Chiapas", Cuicuilco, núm. 62, pp. 149-173.

Wolf, Eric, 1966, Peasants, New Jersey, USA, Prentice Hall. 